\title{
MULTIWAVELENGTH EXPERIMENTS ON AGN:
}

\author{
REVERBERATION EXPERIMENTS
}

\author{
W. WAMSTEKER and R. VIO \\ ESA IUE Observatory, P.O. Box 50727, 28080, Madrid, Spain
}

\begin{abstract}
The nature of the successful reverberation campaigns on variable active galaxies is summarized. A general summary of the completed campaigns is given. A discussion of the results of the first order (CCF) analysis is given and some comments are made on the directions needed to obtain more physcally meaningful solutions to the inversion problem presented by such observations of the sources of activity in the centers of active galaxies
\end{abstract}

Key words: Seyfert I; Ultraviolet ; Multiwavelength; Broad-line-region

\section{Introduction}

Since the original suggestion that the line emission in active galaxies (AGN) is caused by photo-ionization and reverberation will permit to probe the Broad-Line Region (BLR) structure, many observations have been made in the optical, but especially in the UV with the IUE (International Ultraviolet Explorer), towards that end. Many early attempts were defined to try to match the fast variations in Seyfert I galaxies discovered in the X-Ray domain. The original concept that the continuum radiation was correlated over a large range of energies from IR to XRays, motivated this approach. This basic assumption was of course dramatically biased by the very incomplete sampling of the Fourier domain of AGN variation (most X-Ray observations consisted of single observations of a duration of the order of hours) as well as the lack of simultaneous multiwavelength observations. Only in a few cases a different and more systematic approach to these problems was taken such as the long term observational studies by Lloyd (1984) and the long term AGN monitoring at Florida State.

Only after some 7 years of extensive AGN studies with IUE, with the availability of the archival data of IUE, and the recognition that archival studies in astronomy are a respectable activity, was it realized that a major concerted effort of the AGN community would be needed to obtain sufficient observing time to address the variability of, especially Seyfert I galaxies, in a profitable way. A compilation of the results for all 522 active galaxies observed before 1990 with IUE, together with relevant multiwavelength information, is given in the IUE-ULDA Guide No.4 (Courvoisier and Paltani, 1992). Examples illustrating the directions in which progress could be made with the use of IUE archival data as well as dedicated observations with IUE are given in the UV and IR study of F-9 (Clavel, Wamsteker and Glass, 1989) and the UV and optical study of NGC 5548 (Wamsteker et al., 1990).

During a workshop held in Segovia under the auspices of the ESA IUE Obser-

T. J.-L. Courvoisier and A. Blecha: Multi-Wavelength Continuum Emission of AGN, 159-162.

(C) 1994 IAU. Printed in the Netherlands. 
vatory in 1987, a group of AGN researchers agreed to share their resources and attempt to obtain enough observing time both on public and private observatories, to do what could be considered to be a "Reverberation Experiment" in Astrophysics. Under the leadership of a small group, the "Central Committee" (grown somewhat in recent years, but still less than 10 people) a call was issued to the world-wide community and a loosely bound group of scientists, the "AGN Watch" (some 100 AGN researchers), constituted itself with the purpose to support, through their individual specialized contributions, in many different ways the first reverberation experiment. This was done on the intermediate Seyfert I galaxy NGC 5548 in 1989 with an extensive IUE observing run of observations every four days. In parallel with this, the $A G N$ Watch made also a worldwide optical campaign which was performed equally successful (Peterson et al., 1991). The original plans by the $A G N$ Watch were drawn up on the basis of the well documented of variability history of NGC 5548, especially in the UV. The results of this first IUE campaign have been published by (Clavel et al., 1991). It is important to recognize the cooperation of the staff and management of both IUE Observatories, since without their support this first innovative program would not have been completed as successfully as it was.

\section{The reverberation Experiments}

The reasons why the initiative for the reverberation experiments was closely associated with the IUE Space Observatory are:

- The wavelength range accessible with IUE $(115.2 \mathrm{~nm}<\lambda<320 \mathrm{~nm}$, (half of this range in a single exposure) covers important photo ionization diagnostics at different ionization levels, such as Ly $\alpha \lambda 121.6$, CIV $\lambda 154.9$, He II $\lambda 164.0$, CIII] $\lambda 190.9$ and $\mathrm{Mg}$ II $\lambda 279.8$ and is close to the ionizing continuum.

- Variability amplitudes had been found to be larger in the UV than in other wavelength domains.

- The operations mode of IUE would easily allow the introduction of the complex scheduling requirements associated with a regular spacing and frequent return to a specific target.

- The fact that IUE is a facility available to astronomers from all countries made a worldwide coordinated effort easier.

- The IUE orbit and ground control mode allowed uninterrupted scheduling without additional constraints.

- The use of a space observatory removed the problems associated with the terrestrial weather.

- The IUE users had already extensive experience with multiwavelength observations using different observatories and could this way obtain considerable added value through coordinated observations from the $\mathrm{X}$-Rays to the Infrared.

Even before these systematic studies were started the first firm evidence of delays between the variations in the continuum of an active galaxy and the delays 
TABLE I

Results of Major AGN Reverberation Experiments (unit: Days)

\begin{tabular}{|c|c|c|c|c|c|c|c|c|}
\hline \multirow[t]{2}{*}{ Mean sample interval } & \multicolumn{2}{|c|}{$\begin{array}{c}\text { NGC } 4151 \\
3 \text { Days }\end{array}$} & \multicolumn{2}{|c|}{$\begin{array}{c}\text { NGC } 3783 \\
3 \text { Days }\end{array}$} & \multicolumn{2}{|c|}{$\begin{array}{c}\text { NGC } 5548 \\
\text { \& Days }\end{array}$} & \multicolumn{2}{|c|}{$\begin{array}{c}\text { F-9 } \\
60 \text { Days }\end{array}$} \\
\hline & $\Delta \mathrm{T}$ & FWHM & $\Delta \mathrm{T}$ & FWHM & $\Delta \mathrm{T}$ & FWHM & $\Delta \mathrm{T}$ & FWHM \\
\hline$F \lambda 184$ & 1.5 & 22 & $\mathbf{0}$ & 14.2 & $\mathbf{0}$ & 38 & $\mathbf{0}$ & 63 \\
\hline$F \lambda 270$ & & & -1.4 & 18.5 & $\mathbf{0}$ & 40 & & \\
\hline F Opt. & 3.5 & 25 & & & 4 & 42 & 6 & 86 \\
\hline He II $\lambda 164.0$ & & & 0.5 & 11.3 & 10 & 40 & & \\
\hline CIV $\lambda 154.9$ & 5.0 & 26 & 5.5 & 18.6 & 16 & 36 & 200 & 80 \\
\hline$L y \propto \lambda 121.6$ & & & 3.8 & 15.6 & 12 & 34 & 115 & 70 \\
\hline$M g I I \lambda 279.8$ & 5.0 & 23 & 8.5 & 14.7 & $\sim 50$ & 72 & 160 & 90 \\
\hline$H \beta \lambda 486.1$ & & & 7.5 & 40.7 & 18 & 50 & & \\
\hline
\end{tabular}

between the continuum were found for the luminous galaxy Fairall 9, which after its dramatic drop in brightness seen in the UV (Clavel, Wamsteker and Glass, 1989) and X-Rays (Morini et al., 1986), was followed on a semi-regular basis and cross correlation showed significant differences in the times of UV continuum minimum and the times of minimum emission line flux (see table I). The following objects have been subjected to systematic variability monitoring studies:

3C273: UV (Ulrich et al., 1993), Optical, IR, Radio

NGC 5548: $X$-Ray (Clavel et al., 1992), UV (Clavel et al., 1991), Optical (Peterson et al., 1991)

NGC 4151: X-Rays (Perola et al., 1986), UV (Clavel et al., 1990)

NGC 3783: $U V$ (Reichert et al., 1994), Optical/IR (Stirpe et al., 1994).

Pks 2155-304 (Edelson et al., 1994),: X-Ray (Brinkmann et al., 1994), UV (Urry et al., 1993), Optical/IR/Radio (Courvoisier et al., 1994).

Since for 3C273 the emission lines do not vary significantly with the UV continuum (Ulrich et al., 1993) and (O'Brien et al., 1989), and for the BL Lac object Pks 2155-304 (Urry et al., 1993) and (Edelson et al., 1994) the absence of emission lines, precludes the application of reverberation studies, even though the results for these objects are very important for the analysis of the continuum variations in themselves.

\section{Results}

The main results from these campaigns on NGC 4151, NGC 3783 and NGC 5548 are given in table I, together with the earlier results on F-9. Table I gives the time delay $(\Delta T)$ measured from the centroid of the cross correlation function (CCF) of the short wavelengths UV continuum with the parameter given in the first column.

The optical continuum is measured either with the IUE Fine- Error-Sensor or 
as a spectroscopic flux measured near $550 \mathrm{~nm}$. The second parameter in table I (FWHM) indicates the Full- Width-Half-Maximum of the respective CCF. This does not give the accuracy of the delays, but is rather a measure of the correlation length in the lightcurves if the sampling is sufficiently small with respect to the variability timescale. For the case of NGC 5548, also a shorter campaign was made in coordination between IUE and GINGA. It was found that the 2-10 keV X-Rays were within the resolution of the sampling simultaneous, i.e. the delay between the X-Ray continuum and the UV continuum was less than 4 days (Clavel et al., 1992).

The results in Table I show clearly that reverberation experiments were extremely successful and represent a powerful tool to study Active Galaxies. The delays derived are in general much shorter than would be expected from classical central photoionization theory and also acrretion rate changes are imcompatible with the variations shownfor the energy distribution in the "big blue bump". On the other hand, it has become obvious that more sophisticated tools than the CCF alone must be applied to interpret such data properly. The various methods to perform lightcurve inversion are discussed in detail elsewhere in these proceedings by Krolik. It has also become clear that for various well studied objects e.g. NGC4151 (Clavel et al., 1990) and F-9 (Clavel, Wamsteker and Glass, 1989) the emission lines vary rather differently at different velocity ranges. These specific velocity limited variations could make that, although the parameters derived through CCF and MEMECHO methods are of the right order of magnitude for the BLR, a diferent approach might be needed for the analysis of such data sets to acquire a satisfactory understanding of the nature of the BLR. The UV reverberation studies have supplied a completely new tool to study active galaxies, where access to the Ultraviolet wavelength range is vital.

\section{References}

Brinkmann, W., et al., 1994, ApJ, in press

Clavel, J., Wamsteker,W., Glass,I.S., 1989,ApJ, 337, 236

Clavel, J., et al., 1990, MNRAS, 246, 668

Clavel, J., et al., 1991, ApJ, 366, 64

Clavel, J., et al., 1992, ApJ, 393, 113

Courvoisier, T.J.-L., et al., 1994, ApJ, in preparation

Courvoisier, T.J.-L. and Paltani, S., 1992, IUE-ULDA Access Guide \# 4, ESA SP 1153

Edelson, R., et al., 1994, ApJ, in press

Lloyd, C., 1984, MNRAS, 209697

Morini, M., et al., 1986, ApJ, 307, 486

O'Brien, P.T., Zheng, W., Wilson,R., 1989, MNRAS, 240,741

Perola, G. C., et al., 1986, ApJ, 306, 508

Peterson, B.M., et al., 1991, ApJ,368, 119

Reichert, G.A., et al., 1994,ApJ, in press

Stirpe,G.M., et al., Astron. Astrophys.,1994, in press

Ulrich,M.-H., Courvoisier,T.J.-L., Wamsteker, W., 1993, ApJ, 411, 125

Urry, C.M., et al., 1993, ApJ, 411, 614

Wamsteker, W., et al., 1990, ApJ, 354, 446 